\title{
Experimental Investigation of the Plasma-Wall Transition
}

\author{
Tilmann Lunt ${ }^{1}$, Gerd Fussmann ${ }^{1,2}$, and Ole Waldmann ${ }^{2}$ \\ ${ }^{1}$ Humboldt-Universität zu Berlin, Institut für Physik, Newtonstr. 15, 12489, Berlin, Germany and \\ ${ }^{2}$ Max-Planck-Institut für Plasmaphysik, EURATOM Association, Boltzmannstr. 2, 85748 Garching, Germany*
}

\begin{abstract}
We report on measurements of the ion velocity distribution as a function of distance to a target immersed in a magnetized argon plasma. Two situations are investigated, (a): practically the whole plasma streams onto a large target, and (b): the size of the target is significantly smaller than the plasma diameter. The Mach number $M=u / c_{s}$ decreases from $M=1$ at the target surface to values around 0.5 at a typical scale of $\lambda_{a}=20 \mathrm{~mm}$ and $\lambda_{b}=5 \mathrm{~mm}$, respectively. In order to explain these small decay lengths, the measurements of case (a) are compared with a source-diffusion model and those of (b) to Hutchinson's model [1]. In the case of (a) good agreement between modeling and experiment is obtained assuming a low neutral gas temperature. The data in (b) also agrees excellently with modeled profiles, although the large fitting parameter $D=20 \mathrm{~m}^{2} / \mathrm{s}$ indicates that other processes than diffusion contribute significantly to the transport.
\end{abstract}

PACS numbers: 52.25.Xz,52.25.Ya,52.30.-q,52.40.Hf,52.70.-m,52.25.Fi

When modeling the streaming of plasmas with direct wall contact (e.g. the divertor plasma of a fusion device), for the interpretation of the data measured with electrical probes (such as Langmuir or Mach probes), and for the choice of materials relevant to fusion, an understanding of the plasma wall transition is essential. While there are a variety of theoretical models (cf. [2] and references therein) which describe this situation, experimental data is far more rare. So far only investigations at very low densities $\left(n_{e} \leq 10^{15} \mathrm{~m}^{-3}\right)$ in complex magnetic topologies have been performed [3-5]. The only experiment (applying a non-invasive technique) [6] at densities more relevant to fusion research, $n_{e}=10^{18} \mathrm{~m}^{-3}$, finds a clearly subsonic streaming velocity in front of the surface, in contrast to the prediction of Bohm [7]. This letter seeks to clarify the situation.

As a non-invasive diagnostics Laser Induced Fluorescence (LIF) capable of measuring the velocity distribution of the ions in the plasma is the most adequate method to investigate this situation experimentally. First measurements at PSI-2 were carried out in 2003 [8]. At that time, however, the spatial resolution due to the type of laser applied was poor $(\Delta z=50 \mathrm{~mm})$ and the distribution of neutral particles not very clear. Now we are able to report on measurements with a much higher spatial resolution $(\Delta z=0.4 \mathrm{~mm})$ and on an improved modeling. Fig. (1) shows the schematic arrangement of the plasma generator PSI-2 with the LIF diagnostic installed. In PSI-2 magnetized plasmas with direct wall contact, similar to divertor plasmas in fusion devices, can be studied. Details about the PSI-2 plasma are given in [9]. In contrast to fusion devices, however, PSI-2 constitutes a linear arrangement with a much simpler magnetic topology. Besides hydrogen, noble gases are also used as working gases, in particular here argon. Between the heated $\left(T \approx 1500^{\circ} \mathrm{C}\right)$ cylindrical $\mathrm{LaB}_{6}$ cathode

*Electronic address: tilmann.lunt@physik.hu-berlin.de

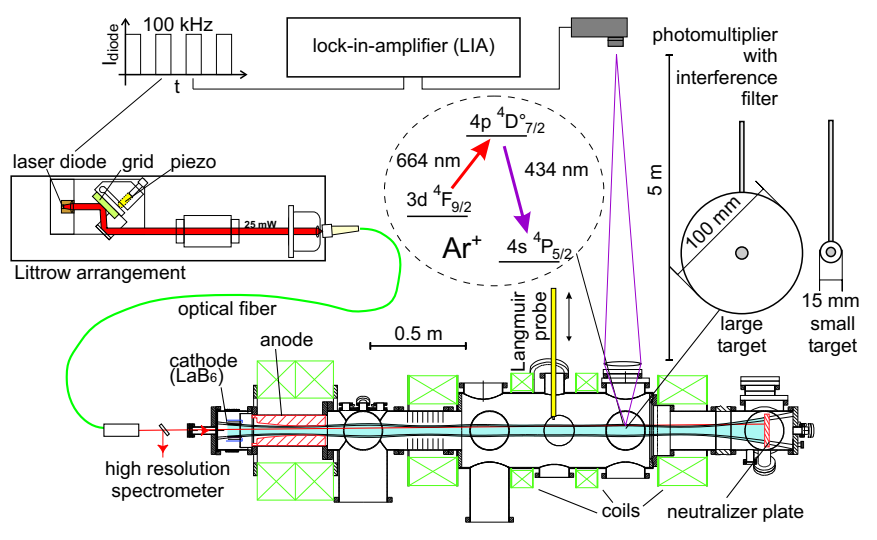

FIG. 1: LIF diagnostic installed in plasma generator PSI-2

and the molybdenum anode, a stationary high current $\left(I_{D} \approx 200 \mathrm{~A}\right)$ arc discharge, fed by a gas inlet $\left(\Phi_{n}=100\right.$ sccm), is sustained. Confined by the axial magnetic field $(|\vec{B}|=85 \mathrm{mT})$ and driven by the pressure gradient it streams towards the neutralizer plate at the end of the device. A great portion of the primary plasma is lost within the hollow anode region, but the fraction escaping from there is amplified due to ionization in a thin cylindrical zone extending over the whole remaining part of the discharge. Due to the shape of the cathode and anode the plasma is primarily maintained within a hollow column $10 \mathrm{~cm}$ in diameter. The radial profile of electron density $n_{e}$ and temperature $T_{e}$, shown in Fig. (2), was measured by means of a Langmuir probe located in the center of the target chamber. The ionization profile $S_{i}=n_{e} n_{n} R_{i}\left(T_{e}\right)$, computed from the local $n_{e}$ and $T_{e}$ values and from the rate coefficient for ionization $R_{i}$ is also shown in the figure (cf. [10]). As both $T_{e}$ and $n_{e}$ are peaked there ionization mainly takes place in the region $r=15 \ldots 30 \mathrm{~mm}$. A finite plasma density outside this source region can only be maintained by means of crossfield diffusion.

The tunable diode laser excites ions in the metastable 


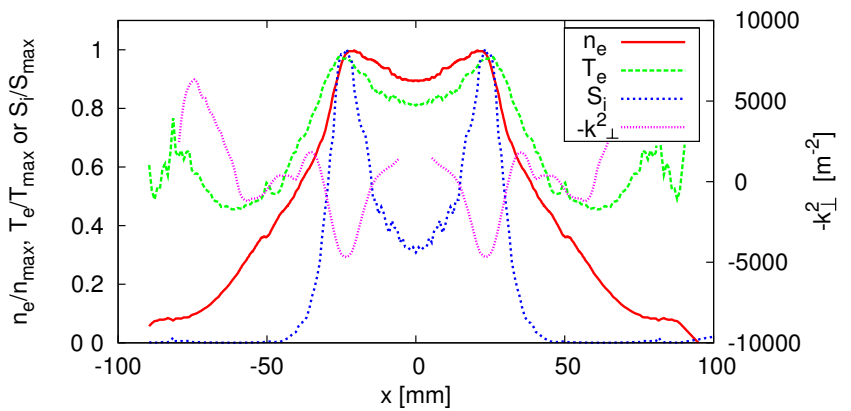

FIG. 2: Typical $n_{e}$ and $T_{e}$ profiles in PSI-2. $S_{i}$ represents source strength due to electron-impact ionization. The quantities are normalized to their peak values $n_{\max }=4.3 \times 10^{18}$ $\mathrm{m}^{-3}, T_{\max }=3.1 \mathrm{eV}$ and $S_{\max }=1.7 \times 10^{22} \mathrm{~m}^{3} \mathrm{~s}^{-1} \cdot k_{\perp}$, a measure for the curvature of the density profile, is computed according to the differential operator in Eq. (1)

level $3 d{ }^{4} F_{9 / 2}$ to the $4 p{ }^{4} D_{7 / 2}$ level (at $\lambda_{0}=664.3698$ $\mathrm{nm})$ causing fluorescence due to the subsequent spontaneous decay to $4 s^{4} P_{5 / 2}$ (lifetime $\tau=1.4 \times 10^{-8} \mathrm{~s}$ ) at $434.8 \mathrm{~nm}$. As the band width of the laser radiation is much smaller than the Doppler width of the transition, only those ions are excited that are in the velocity interval $v_{z} \ldots v_{z}+\Delta v_{z}$, where $v_{z}=c\left(\lambda_{0}-\lambda_{L}\right) / \lambda_{0}$ is the velocity component corresponding to the Doppler shift $\lambda_{L}-\lambda_{0}$. Scanning the laser wavelength $\lambda_{L}$ while detecting the fluorescence allows to measure the ion velocity distribution (ivdf) $f\left(v_{z}\right)$ in the direction of the laser beam.

In order to be able to detect the weak fluorescence signal amidst the very strong background radiation of the plasma it is necessary to apply lock-in-technique (modulation frequency $100 \mathrm{kHz}$ ) and to optimize the optical system. Two aberration-corrected lenses (Apo Tessar 9:900) with a diameter of $\varnothing=100 \mathrm{~mm}$ were used to map the radiation from the plasma to a plane $5 \mathrm{~m}$ away from the target chamber (and from the magnetic coils) where a photomultiplier with a narrow band-width interference filter is located. Its position and its angle of rotation (the transmission of the filter is very sensitive to the incidence angle) can be controlled by means of three manipulators, in order to adjust the distance $|z|$ of the detection volume to the target surface. In front of the photomultiplier an adjustable slit (aperture $\Delta z=4 \mathrm{~mm}$ $\times \Delta y=10 \mathrm{~mm}$ ) is mounted in order to adapt the detection volume to the excitation volume. Since the image is ten times larger than the object, the spatial resolution in axial direction is $\Delta z=0.4 \mathrm{~mm}$.

The laser beam is launched axially into the target chamber where it hits the target, a circular disc made of boron nitride (an insulator) immersed in the plasma. Two different target sizes were used; a 'small' target with $\varnothing=15$ $\mathrm{mm}$ in diameter and a 'large' one with $\varnothing=100 \mathrm{~mm}$. In the center of each target, on both, front and back side, electrical collectors with a diameter of $6 \mathrm{~mm}$ are located. These are used to measure the current-voltage characteristics. The targets therefore also act as Mach probes [1].
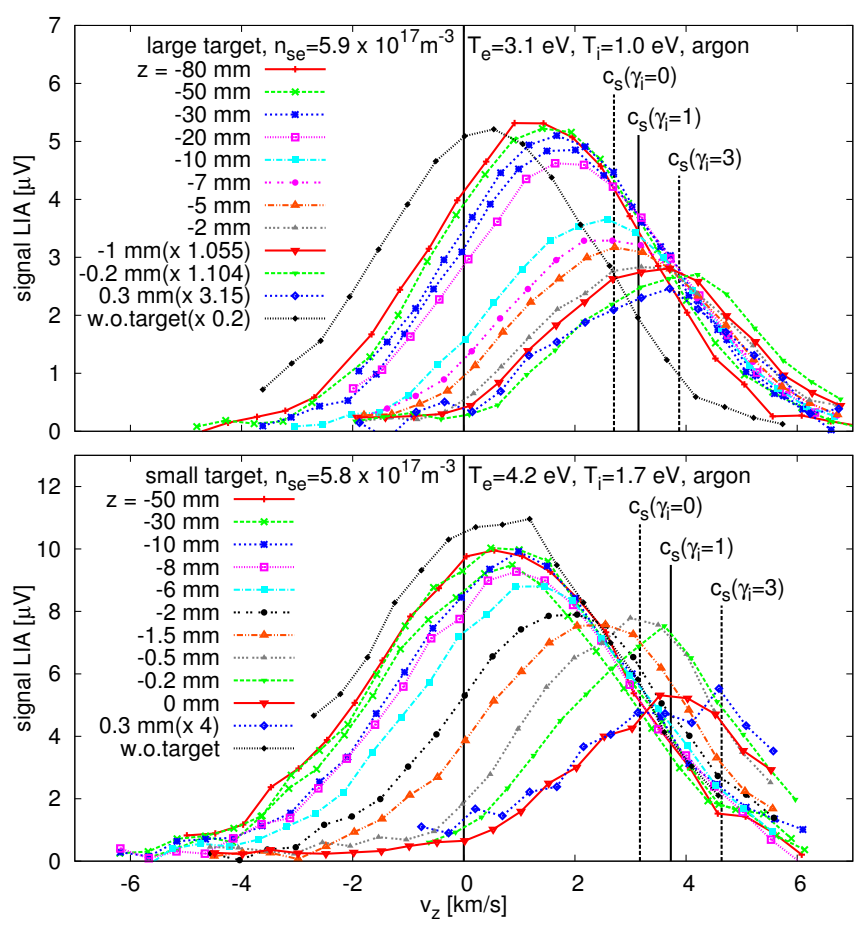

FIG. 3: Measured ivdfs at different axial positions. The sound velocities assuming different values of the ion adiabatic coefficient $\gamma_{i}$ are indicated by the vertical bars.

The LIF measurements carried out at different axial positions are shown in Fig. (3). In order to calibrate the $v_{z}$-axis in Fig. (3) it is necessary to measure the wavelength of the laser very accurately. This was achieved by means of a high resolution Fastie-Ebert spectrometer (UHRS 1500, Sopra) with a focal length of $1.5 \mathrm{~m}$. In order to find the central wavelength, the ivdf was measured at a fixed position in the plasma with the laser launched first in axial and then in counter-axial direction. In order to compensate drifts of the refractive index of the air due to temperature or pressure fluctuations the wavelength of the laser was determined relative to a line from a spectral lamp. The ion temperature evaluated from the ivdf was corrected by $0.5 \mathrm{eV}$ in order to compensate the Zeeman splitting $(\Delta \nu \approx 2.5 \mathrm{GHz})$. Note however, that due to the symmetry of the effect, the mean velocity is not affected. Finally, corrections with respect to the vignetting by the target were made. The correction factors are shown in the legend in parenthesis. For the sake of comparability the ivdf measured when the large target was removed from the plasma is shown reduced by a factor of 5 .

It was found that the ivdfs measured in front of the target are shifted Maxwellian distributions, their shift increasing to the speed of sound when approaching the target. A first important result is this experimental proof of the Bohm criterion [7].

Since the size of the large target exceeds the diameter of the central plasma region, almost all of the plasma streams onto it. It is therefore necessary to set up a model 
for the entire plasma. In order to account for the cylindrical symmetry of the plasma, we write the stationary continuity equation in cylindrical coordinates, assuming cross-field diffusion $\vec{\Gamma}_{\perp}=-D_{\perp} \nabla n$

$$
D_{\perp} \underbrace{\frac{1}{r n} \frac{\partial}{\partial r} r \frac{\partial n}{\partial r}}_{=:-k_{\perp}^{2}}+\nu_{i}=\frac{1}{n} \frac{\partial}{\partial z}(n u)=: \mu,
$$

where quasi-neutrality $n=n_{e}=n_{i}$ and ambipolarity, i.e. equal streaming velocities $u=u_{i}=u_{e}$, were assumed. The differential operator in the first term of (1) can be evaluated for the present density profile shown in Fig. (2). It was found that its value at the position where the LIF measurements were carried out $(r=25 \mathrm{~mm})$, is about $k_{\perp}=66.3 \mathrm{~m}^{-1}$. The ion production frequency $\nu_{i}=R_{i} n_{n}$ is thus reduced effectively by $-D_{\perp} k_{\perp}^{2}$. The stationary one-dimensional continuity and momentum equations for the flux tube at $r=25 \mathrm{~mm}$ in the axial direction then read

$$
\begin{aligned}
\frac{\partial}{\partial z}(n u) & =\mu n=\left(\nu_{i}-k_{\perp}^{2} D_{\perp}\right) n \\
0 & =-\frac{\partial p_{e}}{\partial z}-e n E_{z} \\
m_{i} n u \frac{\partial u}{\partial z} & =-\frac{\partial p_{i}}{\partial z}+e n E_{z}-m_{i} n u\left(\nu_{i}+\nu_{c x}+\nu_{e l}\right)
\end{aligned}
$$

where $E_{z}$ is the electric field and $p_{e, i}$ are the electron and ion pressures respectively. $\nu_{c x}=\sigma_{c x} n_{n} \sqrt{2 T_{i} / m_{i}}$ and $\nu_{e l}=\sigma_{e l} n_{n} \sqrt{2 T_{i} / m_{i}}$ are the frequencies for charge exchange and elastic collisions. We assume $\sigma_{c x}=5.8 \times$ $10^{-19} \mathrm{~m}^{2}[11]$ and $\sigma_{e l}=0.36 \sigma_{c x}$. Rather than discussing the energy equation, electron and ion temperatures $T_{e, i}$ are assumed to be isothermal (and hence the adiabatic coefficients $\gamma_{i}=\gamma_{e}=1$ ), so that $\partial p_{e, i} / \partial z=T_{e, i} \partial n_{e, i} / \partial z$. We then add Eq. (3) and Eq. (4), replace $\partial n / \partial z$ by means of Eq. (2), multiply by $u /\left(n m_{i} c_{s}^{3}\right)$, where $c_{s}=$ $\sqrt{\left(T_{e}+\gamma_{i} T_{i}\right) / m_{i}}$ is the speed of sound, and introduce the Mach number $M=u / c_{s}$ to get the relation

$$
\frac{\partial M}{\partial z}=\frac{\mu}{c_{s}} \frac{1+\alpha M^{2}}{1-M^{2}}, \text { where } \alpha=\frac{\nu_{t}}{\mu},
$$

with the total collision frequency given by $\nu_{t}=\nu_{i}+$ $\nu_{c x}+\nu_{e l}$. The density $n(M)$ as a function of the Mach number can be obtained from (2), determining $\partial n / \partial z$ and then dividing this expression by $n \partial M / \partial z$. Both sides of the equation obtained can be expressed by logarithmic derivatives, so that we find

$$
n(M)=n_{0}\left(1+\alpha M^{2}\right)^{-\frac{\alpha+1}{2 \alpha}}
$$

where $n_{0}$ is the density in the bulk plasma. Knowing the density as a function of $M$ we can directly determine the electric potential $\Phi(M)$ from Boltzmann's relation

$$
\Phi=\frac{T_{e}}{e} \ln (n)
$$

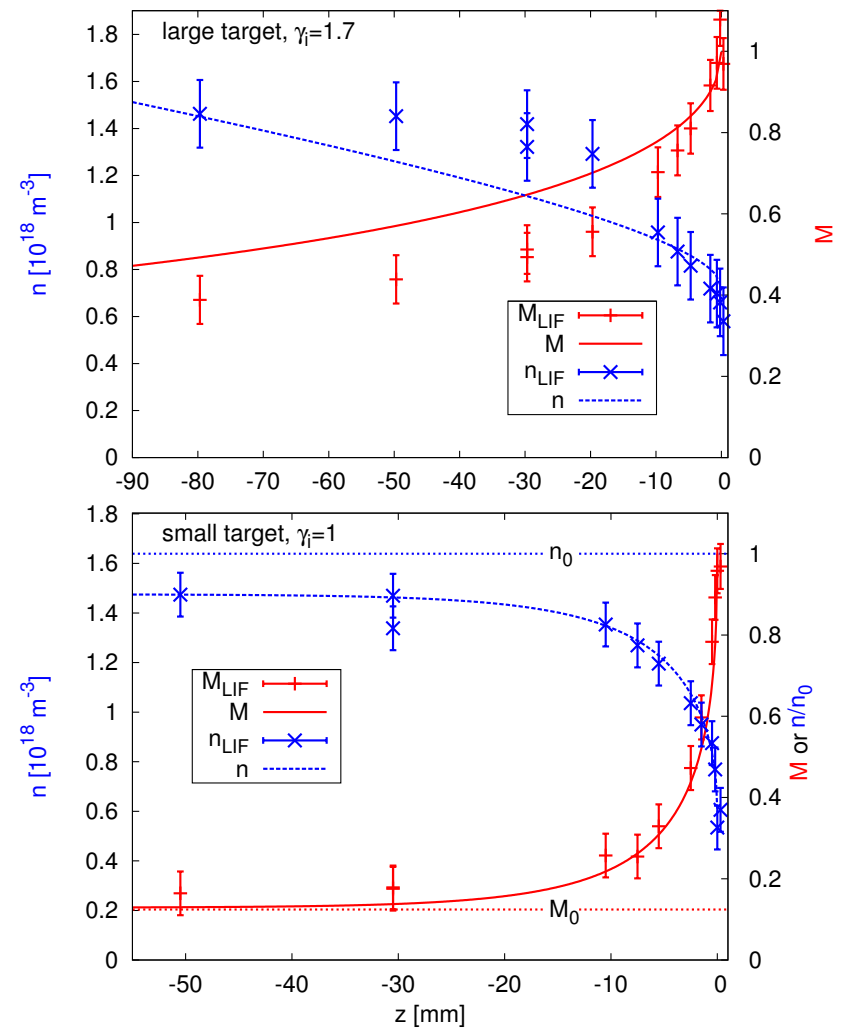

FIG. 4: Measured and fitted values for $M$ and $n$.

which follows from Eq. (3). Note that the ions are accelerated by both, the electric field $\left(e n E_{z}\right)$ and the pressure gradient $\partial p_{i} / \partial z$ in Eq. (4); the ratio of these forces simply being given by the temperature ratio $T_{e} / T_{i} \approx 2$.

Assuming a constant neutral gas density $n_{n}$, i.e. constant $\alpha$ and $\mu$, we can integrate Eq. (5) to obtain an expression for $z(M)$

$$
z(M)=\frac{c_{s}}{\nu_{t}}\left(\frac{1+\alpha}{\sqrt{\alpha}} \arctan (\sqrt{\alpha} M)-M\right)-z_{0} .
$$

$z_{0}$ is chosen such that the Bohm criterion $z(M=1)=0$ is satisfied at the target surface. Axial positions in front of the target then have negative $z$ values.

We have so far disregarded the fact that the target surface, a sink for electrons and ions, is at the same time a strong source of neutral particles. If $T_{e}$ was in the range $10 \mathrm{eV}<T_{e}<15 \mathrm{eV}$ these neutrals would play an important (the dominant) role. Under the present conditions $\left(T_{e}=3.1 \mathrm{eV}\right)$, however, the mean free path of the neutrals (for either ionization or a collision) is at least $\lambda_{n}=u_{n} /\left(n \nu_{t}\right)=12 \mathrm{~cm}$ and can be neglected. Eq. (8) then approximates the experimental data fairly well. Fig. (4) shows the Mach numbers and densities obtained by fitting Maxwellians to the measured ivdfs of Fig. (3). The solid and dashed curves represent Eqs. (8) and (6) with $\alpha=300$ and $\nu_{t}=50 \mathrm{kHz}$. In order to fit the data, a quite high neutral gas density of about $10^{19} \mathrm{~m}^{-3}$, (consistent with the measured pressure only when $T_{n}=380$ 
K) has to be assumed. A simple estimation shows that this can be justified when the energy reflection coefficient $R_{E}$ for the fast neutrals that originate from charge exchange reactions is smaller or equal to $10 \%$. The model also predicts very well the large density increase (of a factor of more than 5), when the target is removed from the plasma, assuming that the neutralizer plate at $z=800 \mathrm{~mm}$ then substitutes the target.

The case of a small target immersed in a magnetized plasma was addressed by Hutchinson [1] in 1988 in order to deduce the Mach number from the ratio of ion saturation currents measured on the two sides of a Mach probe $M_{\infty}=0.4 \ln \left(I_{s 1} / I_{s 2}\right)$. His model takes into account radial particle $\Gamma_{r}=-D \partial n / \partial r$ and momentum $p_{z, r}=-\eta \partial u_{z} / \partial r$ transport, where $\eta=\beta m_{i} n D$. Here $\beta=1$ is chosen.

The profiles for $n$ and $M$ can be computed by integrating numerically Eqs. (14) and (15) in [1]. These were fitted to the measured data in Fig. (4) (bottom). For a diffusion coefficient as high as $D=20 \mathrm{~m}^{2} / \mathrm{s}$ an excellent agreement with the LIF-measurements is obtained. Furthermore, the ratio of the ion saturation currents $I_{s 1} / I_{s 2}=-10.1 \mathrm{~mA} /(-7.3 \mathrm{~mA})$ predicts the unperturbed Mach number $M_{\infty}=0.13$ perfectly. More moderate values around $D=7 \mathrm{~m}^{2} / \mathrm{s}$ are not incompatible with the error bars, but values around $D=1.4 \mathrm{~m}^{2} / \mathrm{s}$ (Bohm diffusion) or $D=0.95 \mathrm{~m}^{2} / \mathrm{s}$ (assumed in the case of the large target) are clearly inconsistent with the data. By including ionization and collision terms in the Hutchinson model this inconsistency cannot be explained either. With the frequencies $\nu_{i}=8 \mathrm{kHz}$ and $\nu_{c x}+\nu_{e l}=10$
$\mathrm{kHz}$ estimated for the measured neutral gas pressure of $p_{n}=24 \mathrm{mPa}$, the computed profiles slightly change, but the very short decay length of the Mach number cannot be reproduced this way.

Assuming that Eq. (7) is valid not only along the field lines but in the whole region, we can determine the radial electric field as $E_{r}=-T_{e} /(e n) \partial n / \partial r$. Besides a poloidal drift, $E_{r}$ also provokes a radial flux of the order

$$
\Gamma_{r}=-\frac{m_{i} T_{e}}{e^{2} B^{2}} \nu_{t} \frac{\partial n}{\partial r} \equiv-D_{E} \frac{\partial n}{\partial r}, D_{E}=4.5 \mathrm{~m}^{2} / \mathrm{s}
$$

which partly explains the observed decay length. This, however, will be addressed in a future publication.

In summary, the Bohm criterion is found to be satisfied in front of (large and small) targets immersed in a plasma. The ions are accelerated by electric and pressure forces over rather short distances $(\leq 2 \mathrm{~cm})$ to achieve sound speed. The findings in [6] were thus not wrong, but the resolution too small to resolve this final acceleration. The measurements can be well reproduced by invoking simple models basing on the momentum balance including radial transport. A extraordinary high radial transport coefficient of $D=20 \mathrm{~m}^{2} / \mathrm{s}$ had to be assumed in order to explain the measurements in front of the small target.

Finally, we would like to thank the whole PSI-2 team, in particular Dr. Bohmeyer, Mr. Mans, and Mr. Mettchen and the mechanical workshop of Humboldt-University.
[1] I.H. Hutchinson, Phys. Rev. A 37, 4358 (1988)

[2] K.U. Riemann, J. Tech. Phys. 41, 89 (2000)

[3] M.J. Goeckner et al., Phys. Fluids B 4, 1663 (1992)

[4] G.D. Severn et al., Phys. Rev. Lett. 90, 145001 (2003)

[5] N. Claire et al., Phys. Plasmas 13, 062103 (2006)

[6] S.L. Gulick et al., J. Nucl. Mater. 176-177, 1059 (1990)

[7] D. Bohm, in The Characteristics of Electrical Discharges in Magnetic Fields (Mc Graw-Hill, New York 1949)
[8] T. Lunt et al., J. Nucl. Mater. 337-339, 201 (2005)

[9] H. Kastelewicz and G. Fussmann, Contrib. Plasma Phys. 44, 352 (2004)

[10] O. Waldmann, et al., Contr. Plas. Phys., 47, 691 (2007)

[11] S.H. Pullins et al., Zeitschr. Phys. Chem., 214, 9, 1279 (2000) 